\title{
The management of clival chordomas: an Italian multicentric study
}

\author{
Luigi Maria Cavallo, MD, PhD,, Diego Mazzatenta, MD, ${ }^{1}$ Elena d'Avella, MD, PhD, ${ }^{1}$ \\ Domenico Catapano, MD, ${ }^{3}$ Marco Maria Fontanella, MD, ${ }^{4}$ Davide Locatelli, MD, ${ }^{5}$ \\ Davide Luglietto, MD, ${ }^{6}$ Davide Milani, MD, ${ }^{7}$ Domenico Solari, MD, PhD, ${ }^{1}$ Marco Vindigni, MD, ${ }^{8}$ \\ Francesco Zenga, MD, ${ }^{9}$ Gianluigi Zona, MD, ${ }^{10}$ and Paolo Cappabianca, MD ${ }^{1}$
}

1Department of Neurosciences and Reproductive and Odontostomatological Sciences, Division of Neurosurgery, University of Napoli "Federico II", Naples; 'Department of Biomedical and Neuromotor Sciences, University of Bologna, IRCCS Institute of Neurological Sciences of Bologna, "Bellaria" Hospital, Bologna; ${ }^{3 D i v i s i o n ~ o f ~ N e u r o s u r g e r y, ~ I R C C S ~ " C a s a ~ S o l l i e v o ~ d e l l a ~}$ Sofferenza", San Giovanni Rotondo; "Division of Neurosurgery, Università degli Studi di Brescia-Spedali Civili, Brescia; ${ }^{5}$ Division of Neurosurgery, Università degli Studi dell'Insubria, Ospedale di Circolo e Fondazione Macchi, Varese; ${ }^{6}$ Division of Neurosurgery, AOU Senese "Le Scotte", Siena; ${ }^{7}$ Division of Neurosurgery, Humanitas Research Hospital, Rozzano; ${ }^{8}$ Neurosurgery Unit, Department of Neurosciences, Santa Maria della Misericordia University Hospital, Udine; ${ }^{9}$ Department of Neuroscience, "Rita Levi Montalcini", Neurosurgery Unit, University of Turin; and ${ }^{10}$ Neurosurgery and Neurotraumatology, San Martino Policlinico Hospital, IRCCS for Oncology and Neurosciences, Genoa, Italy

OBJECTIVE In the last 2 decades, the endoscopic endonasal approach in the treatment of clival chordomas has evolved to be a viable strategy to achieve maximal safe resection of this tumor. Here, the authors present a multicentric national study, intending to analyze the evolution of this approach over a 20 -year time frame and its contribution in the treatment of clival chordomas.

METHODS Clival chordoma cases surgically treated between 1999 and 2018 at 10 Italian neurosurgical departments were included in this retrospective study. Clinical, radiological, and surgical findings, adjuvant therapy, and outcomes were evaluated and compared according to classification in the treatment eras from 1999 to 2008 and from 2009 to 2018.

RESULTS One hundred eighty-two surgical procedures were reviewed, with an increase in case load since 2009. The endoscopic endonasal transclival approach (EETA) was performed in 151 of 182 cases $(83.0 \%)$ and other approaches were performed in 31 cases (17\%). There was an increment in the use of EETA, neuronavigation, and Doppler ultrasound after 2008. The overall postoperative complication rate was $14.3 \%$ (26 of 182 cases) consisting of 9 CSF leaks (4.9\%), 7 intracranial hemorrhages (3.8\%), 5 cases of meningitis $(2.7 \%)$, and 5 cerebral ischemic injuries $(2.7 \%)$. Grosstotal resection (GTR) was achieved in 93 of 182 cases (51.1\%). Extent of resection (EOR) improved in the second era of the study. Signs and/or symptoms at presentation worsened in 27 cases (14.8\%), and the Katz Index worsened in 10 cases (5.5\%). Previous treatment, dural involvement, EETA, and intraoperative Doppler ultrasound correlated with GTR. Patients received adjuvant proton beam radiation in 115 of 182 cases $(63.2 \%)$, which was administered more in the latter era. Five-year progression-free survival (PFS) and overall survival (OS) were $62.3 \%$ and $73.5 \%$, respectively. GTR, EETA, proton beam therapy, and the chondroid subtype correlated with a better survival rate. The mean follow-up was 62 months.

CONCLUSIONS Through multicentric data collection, this study encompasses the largest series in the literature of clival chordomas surgically treated through an EETA. An increase in the use of this approach was found among Italian neurosurgical departments together with an improved extent of resection over time. The satisfactory rate of GTR was marked by low surgical morbidity and the preservation of patient quality of life. Surgical outcome was reinforced, in terms of PFS and OS, by the use of proton beam therapy, which was increasingly performed along the period of study. https://thejns.org/doi/abs/10.3171/2020.5.JNS20925

KEYWORDS chordomas; clivus; endoscopic endonasal approach; multicentric; radiotherapy; surgery; oncology; skull base

ABBREVIATIONS EEA = endoscopic endonasal approach; EETA = endoscopic endonasal transclival approach; GTR = gross-total resection; OS = overall survival; PFS = progression-free survival; $P R=$ partial resection; STR = subtotal resection.

SUBMITTED March 24, 2020. ACCEPTED May 20, 2020.

INCLUDE WHEN CITING Published online September 4, 2020; DOI: 10.3171/2020.5.JNS20925. 


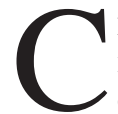
LIVAL chordomas are histologically low-grade tumors, but their behavior can be described as "clinically malignant" because of the diffusely infiltrative growth pattern and high rates of recurrence and tumor-related death..$^{1-5}$ Besides the clivus, the tumor also develops onto surrounding areas of the skull base, infiltrating and eroding the bone and expanding in soft tissues and the intradural compartment. ${ }^{6-9}$ Regardless of the approach, surgical treatment should allow gross-total resection (GTR) of tumor in the soft tissues and extensive bone drilling but probably cannot manage microscopically invisible infiltration into the clivus and adjacent bone. ${ }^{10-13}$ Thus, in spite of aggressive resection, surgical cure of clival chordomas is virtually not achievable from an oncological perspective, and the natural history of the disease remains burdened by overall survival (OS) and progression-free survival (PFS) rates of 70\%-80\% and 50\%-65\% at 5 years, respectively. ${ }^{5,14-16}$

The surgical morbidity and malignant clinical behavior of this tumor have led to a neurosurgical paradigm shift toward a multimodal treatment based on maximally safe resection with the intent of avoiding neurological deficits, followed by radiotherapy. ${ }^{12,17-20}$ Controversies remain regarding the most favorable surgical approach, morbidity, advantages of an aggressive resection in terms of OS, and radiation therapy and its indications for the extent of tumor removal..$^{1,21-23}$

In this setting, the endoscopic endonasal approach (EEA) has been shown in the last 2 decades to be a viable strategy with the possibility of reducing morbidity, as compared to more extensive transfacial or transcranial approaches. ${ }^{18,24-28}$ Nonetheless, there are lesions with lateral extensions in the petrous apex down to the rhinopharynx, the jugular foramen, and the occipital condyles, with or without the intradural component, that are generally considered to expose a limitation of exclusively endoscopic endonasal procedures. . $7,11,19,22$

Here, we present an Italian multicentric study, intending to analyze the evolution of clival chordoma management in a 20-year time frame with an emphasis on surgical treatment. Through a robust statistical analysis of a large number of patients, our study may shed light on the role of the EEA and its impact on improving the outcomes of patients with clival chordomas.

\section{Methods}

Through the compilation of a master database, this multicentric retrospective study included patients harboring a clival chordoma surgically treated between 1999 and 2018. Data were recruited from 10 Italian neurosurgical departments from the public health system. Clinical, radiological, and surgical findings, surgical approach, extent of resection, histopathological diagnosis, adjuvant therapy, and outcome were evaluated. Tumor location was categorized based on anatomical landmarks at the clival bone: upper-clival chordomas involved the sellar floor, dorsum sella, and posterior clinoid processes; middle-clival tumors involved the clival recess of the sphenoid bone; and lower-clival chordomas involved the basilar part of the occipital bone. ${ }^{18}$ Extent of resection was assessed by a neuroradiologist on postoperative MRI as compared to preoperative images and was defined as GTR when no tumor remnant was visible on postoperative MRI, subtotal resection (STR) when more than 50\% of the tumor was resected, and, finally, partial resection (PR) when less than $50 \%$ of tumor removal was achieved.

Data were analyzed and presented as the overall volumes for each of the considered variables. The cohort of patients was classified into two treatment eras, which were then compared. The initial study period was from 1999 to 2008 and the second from 2009 to 2018.

\section{Statistical Analysis}

Continuous variables are presented as the median and range and categorical variables as the absolute and relative frequencies. Comparison of the two eras of surgical treatment was performed using chi-square analysis or ttest. Univariate and multivariate binary logistic regression analyses were performed to study the association between the analyzed variables and extent of resection. Cox regression analysis was performed to study the associations among the analyzed variables, tumor recurrence, and tumor-related death. Survival analyses were performed using the Kaplan-Meier method. A p $<0.05$ was considered to be statistically significant. IBM SPSS version 21 (IBM Corp.) was used for statistical analysis.

\section{Results}

\section{Patient Population and Clinical Data}

We reviewed 78 cases (42.8\%) from Bologna and 104 (57.1\%) from the other centers with an almost homogeneous case load distribution among them. A total of 182 surgical procedures for clival chordomas were performed in 167 patients, with a significantly increased case load in the second era of treatment compared to the first (138:44, $\mathrm{p}<0.001$; Table 1). Patient age ranged from 4 to 85 years (mean 50.2 years), and there was a male predominance (103 male cases, 79 female cases). Sixty-four procedures (64 of $182[35.2 \%]$ ) were performed on recurrent tumors, which had been previously treated as follows: resection (36 of 182 [19.8\%]), resection and adjuvant radiotherapy (26 of 182 [14.3\%]), and radiotherapy only (2 of 182 [1.1\%]). Mean patient age in the study period from 2009 to 2018 was greater than that from 1999 to 2008 ( $\mathrm{p}=0.043$ ), while there was no difference between the two treatment eras with regard to sex $(p=0.71)$ and previous procedures $(p=0.363)$.

The most common symptom at presentation was abducens nerve palsy (113 of 182 [62.1\%]), with concomitant cranial nerve V deficit in 23 of 182 cases (12.6\%). Ophthalmoparesis due to oculomotor and/or trochlear cranial nerve palsy was observed in 72 of 182 cases (39.6\%). In 58 cases $(31.9 \%)$, headache was the presenting symptom. Visual and endocrine disturbances were present in 55 (30.2\%) and 22 (12.1\%) cases, respectively. Lower cranial nerve palsy causing hoarseness/dysphagia was the presenting symptom in 25 of 182 cases (13.7\%), while in 24 cases (13.2\%) it was hemiparesis. At the time of diagnosis, a level 1 status on the Katz Index of Independence in Activities of Daily Living, corresponding to fully active patients, was present in 87 of 182 cases (47.8\%); in 80 of 
TABLE 1. Comparison of clival chordoma characteristics between two treatment eras

\begin{tabular}{|c|c|c|c|c|}
\hline Characteristic & Total & 1999-2008 & 2009-2018 & $\mathrm{p}$ Value \\
\hline No. of cases & 182 & $44(24.2)$ & $138(75.8)$ & $<0.001$ \\
\hline Mean age in yrs & 50.2 & 45.4 & 51.7 & 0.043 \\
\hline \multicolumn{5}{|l|}{ Sex } \\
\hline Male & $103 / 182(56.6)$ & $26 / 103(25.2)$ & $77 / 103(74.8)$ & \multirow{2}{*}{0.71} \\
\hline Female & $79 / 182(43.4)$ & $18 / 79(22.8)$ & $61 / 79(77.2)$ & \\
\hline Previous treatment & $64 / 182(35.2)$ & $18 / 64(28.1)$ & $46 / 64(71.9)$ & 0.363 \\
\hline \multicolumn{5}{|l|}{ Preop level of autonomy in daily activities } \\
\hline Complete autonomy & $87 / 182(47.8)$ & $49 / 87(56.3)$ & $38 / 87(43.7)$ & \multirow{3}{*}{0.182} \\
\hline Partial autonomy & $80 / 182(44.0)$ & $51 / 80(63.8)$ & $29 / 80(36.3)$ & \\
\hline Occasionally or absolutely dependent & 15/182 (8.2) & $8 / 15(53.3)$ & $7 / 15(46.7)$ & \\
\hline \multicolumn{5}{|l|}{ Approach } \\
\hline EETA & $151 / 182(83.0)$ & 28/151 (18.5) & $123 / 151(81.5)$ & \multirow{2}{*}{$<0.001$} \\
\hline Other approaches & $31 / 182(17.0)$ & $16 / 31(51.6)$ & $15 / 31(48.4)$ & \\
\hline \multicolumn{5}{|l|}{ Intraop tools } \\
\hline Neuronavigation & 138/182 (75.8) & $32 / 138(23.2)$ & 106/138 (76.8) & 0.042 \\
\hline Doppler ultrasound & 101/182 (55.5) & 21/101 (20.8) & 78/101 (77.2) & 0.032 \\
\hline Neuromonitoring & $53 / 182(29.1)$ & $23 / 53(43.4)$ & $30 / 53(56.6)$ & 0.156 \\
\hline \multicolumn{5}{|l|}{ EOR } \\
\hline GTR & 93/182 (51.1) & $12 / 93(12.9)$ & $81 / 93(87.1)$ & \multirow{2}{*}{$<0.001$} \\
\hline STR or PR & $89 / 182(48.9)$ & $32 / 89(36.0)$ & $57 / 89(64.0)$ & \\
\hline Postop complication & 26/182 (14.3) & $11 / 26(42.3)$ & $15 / 26(57.7)$ & 0.607 \\
\hline \multicolumn{5}{|l|}{ Postop level of autonomy in daily activities } \\
\hline Complete autonomy & $155 / 182(85.2)$ & $64 / 155(41.3)$ & $91 / 155(58.7)$ & \multirow{3}{*}{0.144} \\
\hline Partial autonomy & 16/182 (8.8) & $9 / 16(56.3)$ & $7 / 16(43.7)$ & \\
\hline Occasionally or absolutely dependent & $11 / 182(6.0)$ & $4 / 11(36.4)$ & $7 / 11(63.6)$ & \\
\hline \multicolumn{5}{|l|}{ RT } \\
\hline Proton beam & $115 / 182(63.2)$ & 27/115 (23.5) & $88 / 115$ (76.5) & \multirow{2}{*}{$<0.001$} \\
\hline Other modalities & $26 / 182(14.3)$ & $11 / 26(42.3)$ & $15 / 26(57.7)$ & \\
\hline Tumor recurrence & 49/182 (26.9) & $13 / 49(26.5)$ & $36 / 49(73.5)$ & 0.421 \\
\hline Tumor-related death & $44 / 182(24.2)$ & $18 / 44(40.9)$ & 26/44 (59.1) & 0.395 \\
\hline
\end{tabular}

182 cases $(44.0 \%)$ the patients presented with partial autonomy (Katz Index level 2), and in 15 of 182 cases (8.2\%) the Katz Index level was $\leq 3$ (patient not capable of performing normal activities or unable to function without assistance). ${ }^{29}$ The presenting Katz Index did not differ between the two treatment eras $(\mathrm{p}=0.182)$.

\section{Radiological and Surgical Data}

Based on their location, tumors were defined as upper(87 of 182 [47.8\%]), middle- (17 of 182 [9.3\%]), lower- (39 of 182 [21.4\%]), or holo-clival (39 of 182 [21.4\%]). Regarding extension, 44 of 182 clival chordomas $(24.2 \%)$ were completely extradural; in 60 of 182 cases $(33.0 \%)$ there was evidence of dural infiltration, and in 78 of 182 cases (42.9\%) tumors showed intradural invasion. Eighty tumors (44.0\%) extended laterally to the internal carotid artery. Maximal tumor diameter (range 1.0-7.5 cm, mean $3.5 \mathrm{~cm}$ ) was $<3 \mathrm{~cm}$ in 54 of 182 cases (29.7\%) and $\geq 3 \mathrm{~cm}$ in 128 of 182 cases $(70.3 \%)$.
An endoscopic endonasal transclival approach (EETA) was performed for the removal of 151 clival chordomas $(83.0 \%)$. The remaining 31 surgical treatments $(17 \%)$ consisted of 13 transcranial approaches $(7.1 \% ; 1$ retrosigmoid, 1 suboccipital, 7 pterional, 2 subfrontal, 1 orbitozygomatic, and 1 subtemporal approach), 7 microsurgical transsphenoidal approaches (3.8\%), 6 combined procedures (3.3\%; 1 EETA and transoral approach, 1 transcranial far lateral and transoral approach, 3 EETA and pterional approach, and 1 EETA and Le Fort I approach), 4 transoral approaches $(2.2 \%)$, and 1 transfacial transmaxillary approach $(0.5 \%)$. There was a significant increase in the use of the EETA and expansion of its indications for middle- and lower-clival chordomas $(\mathrm{p}<0.05)$ between the two treatment eras (Fig. 1). In the first period of study, the EETA was used for the removal of 19 of 26 upperclival chordomas (73.1\%), 1 of 4 middle-clival chordomas (25.0\%), 2 of 7 lower-clival chordomas (28.6\%), and 2 of 8 holo-clival tumors (25.0\%). In the second era of treatment, 


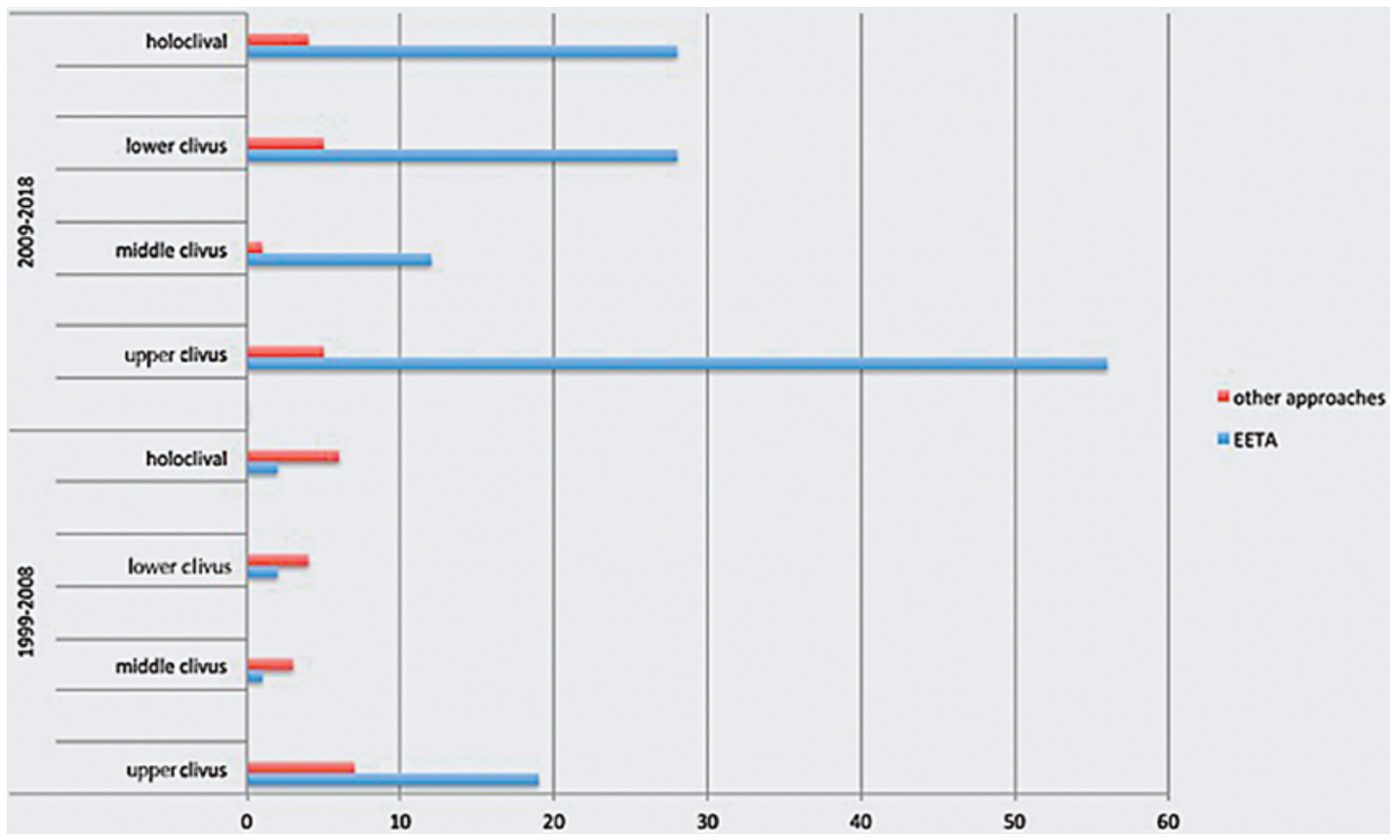

FIG. 1. Stratifying the use of the EETA between the eras of treatment, it is clearly visible that before 2009 this approach was reserved mainly for the removal of upper-clival chordomas. Later, it became the most used approach regardless of tumor location. Figure is available in color online only.

the number of EETAs performed for chordomas localized at the upper third of the clivus was 56 of 61 (91.8\%); at the middle third, 12 of 13 (92.3\%); at the lower third, 28 of 32 (87.5\%); and for holo-clival lesions, 28 of 31 (90.3\%). The number of EETAs performed for primary versus previously treated lesions was not significantly different. There was no significant correlation among the choice of the approach, tumor dimension, or extension.

In the whole series, neuronavigation was used in 138 of 182 procedures (75.8\%), microvascular Doppler ultrasound in 101 of 182 cases (55.5\%), and neuromonitoring in 53 of 182 (29.1\%). There was a significant increase in the use of neuronavigation $(\mathrm{p}=0.042)$ and microvascular Doppler ultrasound $(\mathrm{p}=0.032)$ between the two treatment eras.

The overall rate of postoperative complications was $14.3 \%$ (26 adverse events in 182 surgical procedures), and the complications consisted of 9 CSF leaks (4.9\%),
7 intracranial hemorrhages $(3.8 \%), 5$ cases of meningitis (2.7\%), and 5 cerebral ischemic injuries (2.7\%; Table 2). CSF leaks occurred in 7 tumors with intradural extension (7 of 78 [9.0\%]) and 2 clival chordomas infiltrating the dura (2 of 60 [3.3\%]), but never in extradural tumors. None of the considered variables significantly correlated to the incidence of postoperative complications; their frequency was similar between eras $(\mathrm{p}=0.607)$. Histopathological diagnosis showed 125 classic chordomas $(68.7 \%)$, 47 chondroid subtypes (25.8\%), and 10 dedifferentiated tumors (5.5\%).

\section{Extent of Resection}

In the present series, GTR was achieved in 93 cases (51.1\%), STR in 64 (35.2\%), and PR in 25 (13.7\%), including 7 biopsies. Extent of resection improved in the second study period, after 2008 ( $\mathrm{p}<0.001$ ). GTR was achieved through an EETA in 87 of 151 cases (57.6\%) and through

TABLE 2. Postoperative complication rates divided by surgical approach, treatment era, and extent of resection

\begin{tabular}{|c|c|c|c|c|c|c|c|}
\hline \multirow[b]{3}{*}{$\begin{array}{c}\text { Postop } \\
\text { Complication }\end{array}$} & \multirow{3}{*}{$\begin{array}{c}\text { Total } \\
(26 / 182 \\
[14.3])\end{array}$} & \multicolumn{4}{|c|}{ Surgical Approach } & \multirow{2}{*}{\multicolumn{2}{|c|}{ EOR }} \\
\hline & & \multicolumn{2}{|c|}{ EETA $(n=151)$} & \multicolumn{2}{|c|}{ Other $(n=31)$} & & \\
\hline & & $\begin{array}{c}1999-2008 \\
(n=28)\end{array}$ & $\begin{array}{c}2009-2018 \\
(n=123)\end{array}$ & $\begin{array}{c}1999-2008 \\
(n=16)\end{array}$ & $\begin{array}{c}2009-2018 \\
(n=15)\end{array}$ & $\begin{array}{c}\text { GTR } \\
(n=93)\end{array}$ & $\begin{array}{l}\text { STR/PR } \\
(n=89)\end{array}$ \\
\hline CSF leakage & $9 / 182(4.9)$ & $2 / 28(7.1)$ & $5 / 123(4.1)$ & $2 / 16(12.5)$ & & $4 / 93(4.3)$ & $5 / 89(5.6)$ \\
\hline Intracranial hemorrhage & $7 / 182(3.8)$ & $2 / 28(7.1)$ & $4 / 123(3.3)$ & $1 / 16(6.3)$ & & $3 / 93(3.2)$ & $4 / 89(4.5)$ \\
\hline Meningitis & $5 / 182(2.7)$ & $1 / 28(3.6)$ & $3 / 123(2.4)$ & $1 / 16(6.3)$ & & $3 / 93(3.2)$ & $2 / 89(2.2)$ \\
\hline Cerebral ischemic injury & $5 / 182(2.7)$ & $2 / 28(7.1)$ & $2 / 123(1.6)$ & & $1 / 15(6.7)$ & $2 / 93(2.2)$ & $3 / 89(3.4)$ \\
\hline
\end{tabular}

$\mathrm{n}=$ number of cases.

Values expressed as number/total (\%). 
TABLE 3. Multivariate analysis to assess prognostic factors for gross-total tumor removal

\begin{tabular}{|c|c|c|c|c|}
\hline \multirow[b]{2}{*}{ Variable } & \multirow[b]{2}{*}{ OR } & \multicolumn{2}{|c|}{$95 \% \mathrm{Cl}$} & \multirow[b]{2}{*}{ p Value } \\
\hline & & Lower & Upper & \\
\hline Age & 1.018 & 0.995 & 1.042 & 0.130 \\
\hline \multicolumn{5}{|l|}{ Previous treatment } \\
\hline No & Reference & & & \\
\hline Yes & 0.0520 & 0.171 & 0.907 & 0.029 \\
\hline \multicolumn{5}{|l|}{ Location } \\
\hline Holo-clival & Reference & & & \\
\hline Upper-clival & 1.203 & 0.474 & 3.053 & 0.697 \\
\hline Middle-clival & 6.548 & 1.256 & 34.150 & 0.080 \\
\hline Lower-clival & 1.480 & 0.474 & 4.619 & 0.500 \\
\hline \multicolumn{5}{|l|}{ Max tumor diameter } \\
\hline$<3 \mathrm{~cm}$ & Reference & & & \\
\hline$\geq 3 \mathrm{~cm}$ & 0.683 & 0.255 & 1.834 & 0.450 \\
\hline \multicolumn{5}{|l|}{ Relationship to dura } \\
\hline Extradural extension & Reference & & & \\
\hline Dural infiltration & 0.228 & 0.037 & 0.406 & 0.008 \\
\hline Intradural extension & 0.123 & 0.076 & 0.683 & 0.001 \\
\hline \multicolumn{5}{|l|}{ Relationship to ICA } \\
\hline Medial & Reference & & & \\
\hline Lateral & 1.164 & 0.491 & 2.760 & 0.730 \\
\hline \multicolumn{5}{|l|}{ Approach } \\
\hline EETA & 5.773 & 1.591 & 20.943 & 0.008 \\
\hline Other & Reference & & & \\
\hline \multicolumn{5}{|l|}{ Intraop tools } \\
\hline Neuronavigation & 0.177 & 0.026 & 1.200 & 0.076 \\
\hline Neuromonitoring & 0.350 & 0.100 & 1.225 & 0.100 \\
\hline Doppler ultrasound & 0.258 & 0.097 & 0.684 & 0.006 \\
\hline
\end{tabular}

other approaches in 6 of 31 cases (19.4\%). We found a significant increase $(\mathrm{p}<0.05)$ in the efficacy of the EETA over time: GTR was obtained in 12 of 87 cases (13.8\%) until 2008 and in 75 of 87 clival chordomas (86.2\%) since 2009. Demographic (age), clinical (previous treatment of any type), radiological (clival location, dural involvement, lateral extension beyond the internal carotid artery, maximal diameter), and surgical (approach, use of intraoperative tools) parameters were included in the univariate and multivariate analysis of prognostic factors for GTR in the overall series. The results revealed that previous treatments (OR 0.0520, 95\% CI 0.171-0.907, $\mathrm{p}=0.029)$, dural infiltration (OR 0.228, 95\% CI 0.037-0.406, p = 0.008), intradural extension (OR 0.123, 95\% CI 0.076-0.683, $\mathrm{p}=$ 0.001 ), and the use of intraoperative Doppler ultrasound (OR 0.258, 95\% CI $=0.097-0.684, \mathrm{p}=0.006)$ were verified as negative predictors for GTR. We found a favorable association between the EEA (OR 5.773, 95\% CI 1.59120.943, $\mathrm{p}=0.008$ ) and GTR (Table 3).

\section{Adjuvant Radiotherapy}

One hundred forty-one surgically treated tumors (77.5\%) were targeted with postoperative radiotherapy.
Among them, 81.6\% (115 of 141) received high-energy proton beam therapy, $8.5 \%$ (12 of 141) received photon therapy, $5.7 \%$ (8 of 141) were treated with a combination of photons and protons, and 4.3\% (6 of 141) were treated with Gamma Knife radiosurgery. Adjuvant radiation was administered more frequently in the latter era, with an increased number of patients undergoing proton beam therapy as compared to those in the first study period ( $\mathrm{p}<$ 0.001 ). Radiotherapy was used with no statistically significant difference $(\mathrm{p}=0.83)$ after GTR (70 of 141 [49.6\%]) and STR/PR (71 of 141 [50.3\%]). Radiation therapy toxicity was registered in 7 of 141 cases (5.0\%): brainstem ischemic injury, dysphagia, temporal lobe radionecrosis, sinusitis, tinnitus, amaurosis, and lower-limb paraparesis. This information was not available in 78 of the 141 cases (55.3\%).

\section{Follow-Up}

The mean follow-up for the entire series calculated from the index surgery was 62 months (range 1-264 months). To evaluate how surgical treatments, either the EETA or other approaches, impacted patient quality of life, we assessed the Katz Index and clinical outcome at 3 months after surgery. The patients' level of independence in activities of daily living improved in 85 of 182 cases (46.7\%), remained unchanged in 87 of 182 cases (47.8\%), and worsened in 10 of 182 cases (5.5\%). For each sign or symptom at presentation, the clinical outcome was as follows: headache improved or remained unchanged in 54 of 182 cases (29.7\%) and worsened in 4 of $182(2.2 \%)$; lower cranial nerve palsy causing hoarseness/dysphagia improved or remained unchanged in 23 of 182 cases $(12.6 \%)$ and worsened in 2 of $182(1.1 \%)$; visual function worsened in 4 of 182 cases (2.2\%) and improved or remained stable in 51 of 182 cases (28.0\%); endocrine disturbances improved or remained unchanged in 18 of 182 cases $(98.9 \%)$ and worsened in 4 of $182(2.2 \%)$; cranial nerve V palsy improved or remained unchanged in 21 of 182 cases (11.5\%) and worsened in 2 of 182 cases (1.1\%); ophthalmoparesis was recorded as improved or unchanged in 62 of 182 cases (34.1\%) and as worsened in 10 of 182 (5.5\%); hemiparesis improved or remained unchanged in 23 of 182 cases $(12.6 \%)$ and worsened in 1 case only $(0.5 \%)$. During the follow-up, a total of 49 tumor recurrences $(26.9 \%)$ were observed, with a mean 5-year PFS rate of $62.3 \%$. In 44 cases $(24.2 \%)$, patients died from their disease, yielding a mean 5-year OS rate of $73.5 \%$. There was no significant difference in patients' Katz Index at follow-up $(p=0.144)$, recurrence rate $(\mathrm{p}=0.421)$, and tumor-related death $(\mathrm{p}=$ 0.395 ) between the two treatment eras.

In a multivariate analysis encompassing all the clival chordomas in this series, classic (OR 3.842, 95\% CI 1.282$11.519, \mathrm{p}=0.016)$ and dedifferentiated (OR $11.655,95 \% \mathrm{CI}$ 1.614-84.181, $\mathrm{p}=0.015$ ) histopathological subtypes were verified as poor prognostic factors for tumor recurrence, while adjuvant proton beam radiation (OR $0.265,95 \%$ CI $0.091-0.776, \mathrm{p}=0.015$ ) was a protective independent factor (Table 4). EETA (OR 0.147, 95\% CI 0.023-0.950, $\mathrm{p}=$ $0.044)$, GTR (OR 0.342, 95\% CI 0.129-0.901, $\mathrm{p}=0.030$ ), and adjuvant proton beam radiation (OR $0.121,95 \% \mathrm{CI}$ $0.035-0.414, \mathrm{p}=0.001)$ were independent favorable indi- 
TABLE 4. Multivariate analysis to assess prognostic factors for tumor recurrence

\begin{tabular}{|c|c|c|c|c|}
\hline \multirow[b]{2}{*}{ Variable } & \multirow[b]{2}{*}{ OR } & \multicolumn{2}{|c|}{$95 \% \mathrm{Cl}$} & \multirow[b]{2}{*}{$p$ Value } \\
\hline & & Lower & Upper & \\
\hline Age & 0.982 & 0.957 & 1.008 & 0.177 \\
\hline \multicolumn{5}{|l|}{ Previous treatment } \\
\hline No & Reference & & & \\
\hline Yes & 0.747 & 0.274 & 2.038 & 0.568 \\
\hline \multicolumn{5}{|l|}{ Relationship to dura } \\
\hline Extradural extension & Reference & & & \\
\hline Dural infiltration & 2.137 & 0.231 & 19.767 & 0.503 \\
\hline Intradural extension & 7.211 & 0.879 & 59.180 & 0.066 \\
\hline \multicolumn{5}{|l|}{ Approach } \\
\hline EETA & Reference & & & \\
\hline Other & 1.398 & 0.337 & 5.796 & 0.644 \\
\hline \multicolumn{5}{|l|}{ EOR } \\
\hline GTR & Reference & & & \\
\hline STR/PR & 1.604 & 0.614 & 4.189 & 0.335 \\
\hline \multicolumn{5}{|l|}{ Histopathology } \\
\hline Chondroid & Reference & & & \\
\hline Classic & 3.842 & 1.282 & 11.519 & 0.016 \\
\hline Dedifferentiated & 11.655 & 1.614 & 84.181 & 0.015 \\
\hline \multicolumn{5}{|l|}{ RT } \\
\hline No RT & Reference & & & \\
\hline Proton beam & 0.265 & 0.091 & 0.776 & 0.15 \\
\hline Other modalities & 1.065 & 0.231 & 4.904 & 0.936 \\
\hline
\end{tabular}

Boldface type indicates statistical significance.

cators of OS (Table 5). Kaplan-Meier analysis considering the whole series estimated increased 5-year PFS and OS rates when patients received proton beam radiation compared to other modalities or no adjuvant radiotherapy (Fig. 2). Regarding the degree of resection, patients with GTR had longer PFS and OS than patients with other degrees of resection (Fig. 3), with a significant benefit when surgery was followed by proton beam radiation therapy.

\section{Discussion}

Herein, we present the results of a multicentric study encompassing, to the best of our knowledge, the largest series of EETAs for clival chordomas (151 surgical procedures). The study period from 1999 to 2018, which entailed the expansion of EEAs and the refinement of radiation modalities, allowed us to analyze the evolution of chordoma management and its impact on patient outcomes. ${ }^{4,8,9,12,24,27,28}$ The satisfactory rate of GTR (87 of 151 [57.6\%]) achieved through the EETA, together with the low rate of postoperative complications (21 of 151 [13.9\%]), the low rate of clinical worsening (13 of 151 [8.6\%]), and the minimal reduction in patients' autonomy (4 of 151 [2.6\%]), confirms the efficacy and safety of this approach for the treatment of clival chordomas. The results of our survival analysis (including data from both the EETAs and other approaches), in which 5-year PFS and OS were $62.3 \%$ and
TABLE 5. Multivariate analysis to assess prognostic factors for OS

\begin{tabular}{|c|c|c|c|c|}
\hline \multirow[b]{2}{*}{ Variable } & \multirow[b]{2}{*}{ OR } & \multicolumn{2}{|c|}{$95 \% \mathrm{Cl}$} & \multirow[b]{2}{*}{$p$ Value } \\
\hline & & Lower & Upper & \\
\hline Age & 0.994 & 0.962 & 1.027 & 0.725 \\
\hline \multicolumn{5}{|l|}{ Previous treatment } \\
\hline No & Reference & & & \\
\hline Yes & 2.358 & 0.822 & 6.762 & 0.110 \\
\hline \multicolumn{5}{|l|}{ Approach } \\
\hline EETA & 0.147 & 0.023 & 0.950 & 0.044 \\
\hline Other & Reference & & & \\
\hline \multicolumn{5}{|l|}{ EOR } \\
\hline GTR & 0.342 & 0.129 & 0.901 & 0.030 \\
\hline STR/PR & Reference & & & \\
\hline \multicolumn{5}{|l|}{ Max tumor diameter } \\
\hline$<3 \mathrm{~cm}$ & Reference & & & \\
\hline$\geq 3 \mathrm{~cm}$ & 0.683 & 0.255 & 1.834 & 0.450 \\
\hline \multicolumn{5}{|l|}{ Histopathology } \\
\hline Chondroid & Reference & & & \\
\hline Classic & 1.281 & 0.284 & 5.770 & 0.747 \\
\hline Dedifferentiated & 8.603 & 0.715 & 103.477 & 0.090 \\
\hline \multicolumn{5}{|l|}{ RT } \\
\hline No RT & Reference & & & \\
\hline Proton beam & 0.121 & 0.035 & 0.414 & 0.001 \\
\hline Other modalities & 1.044 & 0.272 & 4.001 & 0.950 \\
\hline
\end{tabular}

Boldface type indicates statistical significance.

$73.5 \%$, respectively, enforce the concept that the optimal management of clival chordoma should be based on surgical removal to the extent of a macroscopically free surgical field, followed by adjuvant radiotherapy in order to achieve control of the diffusively infiltrative microscopic bony disease. ${ }^{14,15,18,30}$

We found patients undergoing endoscopic procedures to outnumber those operated on through other approaches, reflecting the Italian multicentric trend over time of the preferred surgical option for clival chordoma, with increasing indications and satisfactory outcomes. Since 1966, the transsphenoidal approach has been proposed for these tumors, with the natural nasal corridor advocated as the most direct route to the clivus. ${ }^{31,32}$ With the advent of endoscopic technology and the development of extended approaches, the transsphenoidal route has been increasingly implemented in the treatment of clival chordomas. ${ }^{21,27,33-37}$ In comparing the indication based on tumor location, we observed that in the first surgical era until 2008, the EETA was mainly reserved for upper-clival chordomas; later, it progressively replaced other approaches to remove tumors of the middle and inferior third of the clivus. In the present series, tumor location did not affect the extent of resection obtained through the EETA, unlike in previous reports noting how tumors in the inferior clivus tended to remain. ${ }^{19,27,28}$ We found no statistically significant differences $(p=0.073)$ in the GTR rate among endoscopically treated upper- (40 of 75 [53.3\%]), middle- (11 of 13 

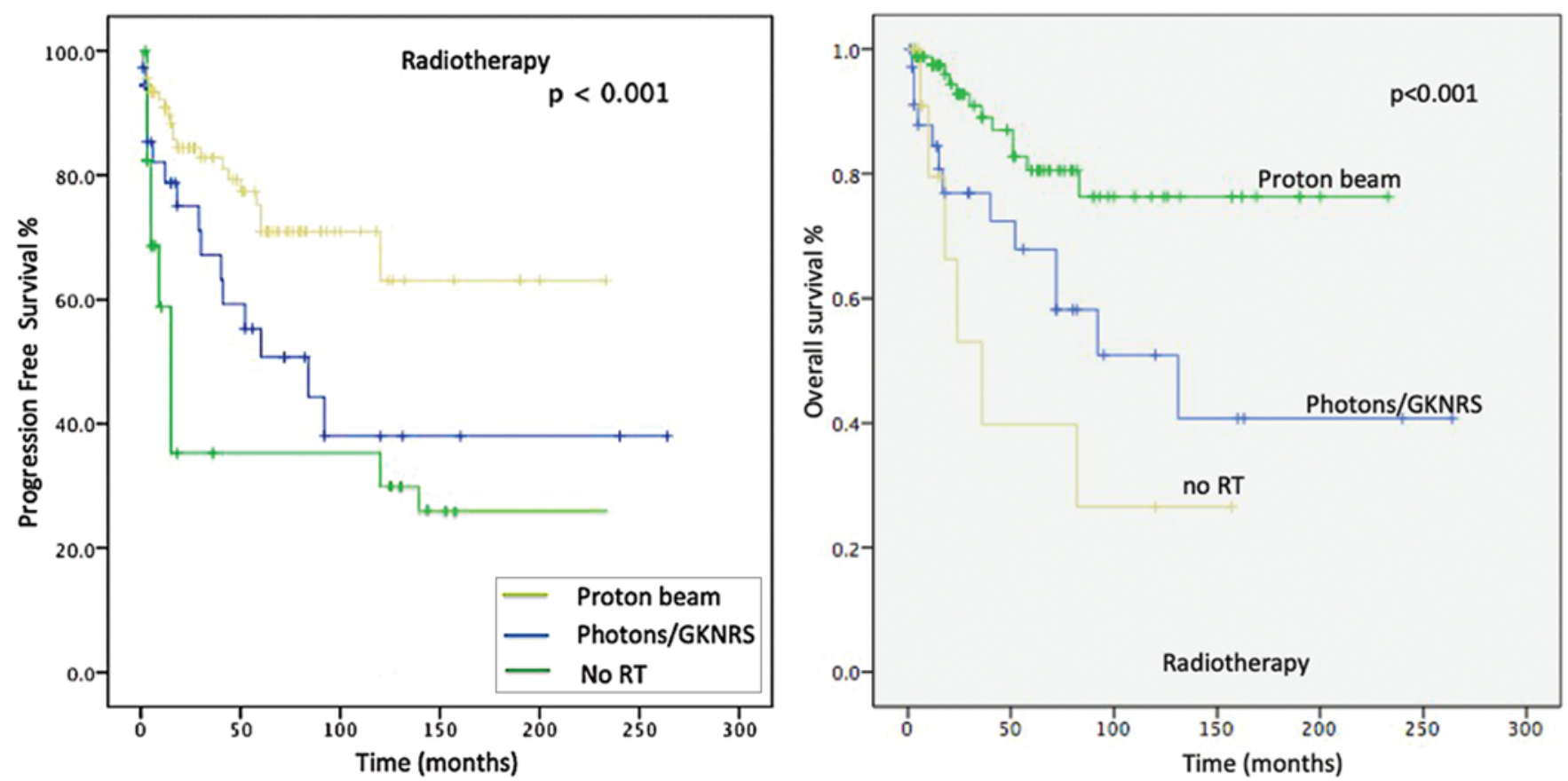

FIG. 2. Kaplan-Meier curves for survival rate associated with adjuvant radiation therapy. PFS (left) and OS (right) rates increased for patients who received adjuvant proton beam therapy, compared to those who received other radiation modalities or none. GKNRS = Gamma Knife radiosurgery. Figure is available in color online only.

[84.6\%]), lower- (19 of 30 [63.3\%]), and holo-clival (17 of 30 [56.6\%]) chordomas. This is probably due to the fact that refinement of the surgical technique and increased experience reduce the intrinsic difficulty of tumor removal

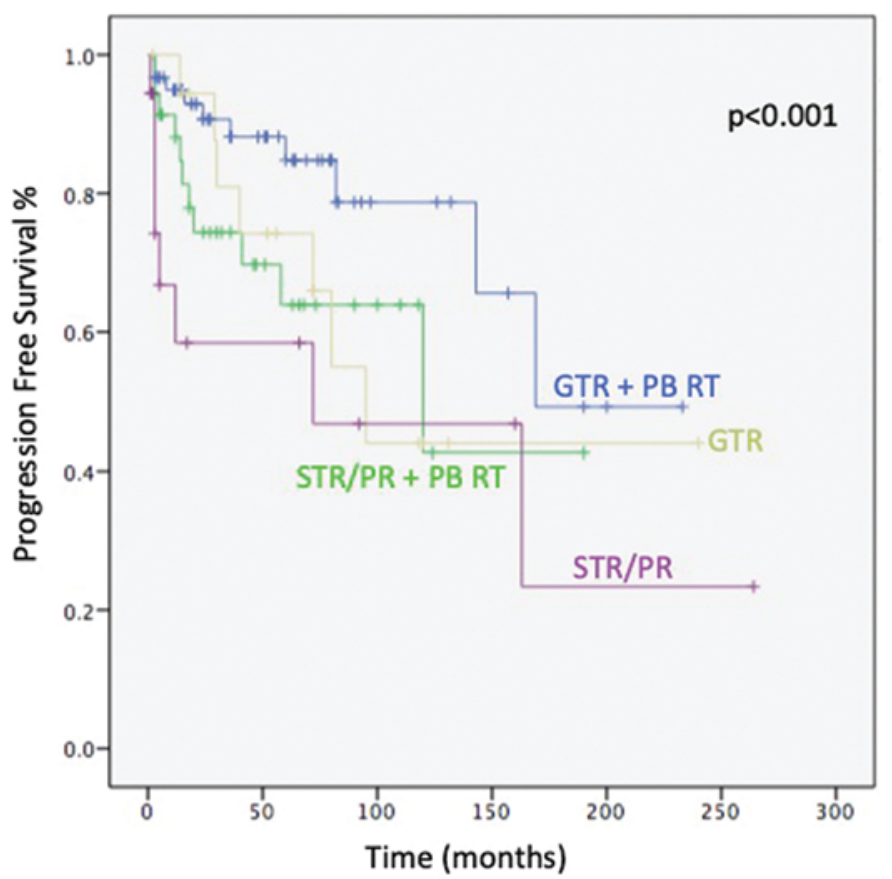

at the anatomical caudal limit of the EEA, which is dense with the critical neurovascular structures of the craniovertebral junction. On the other hand, the relative low rate of GTR among endoscopically treated upper-clival chordo-

FIG. 3. Kaplan-Meier curves of survival rates associated with extent of resection and radiation therapy. PFS (left) and OS (right) increased for patients who underwent GTR and proton beam therapy (PB RT), compared to those who underwent GTR without adjuvant radiation therapy and those who underwent STR/PR with or without proton beam radiation. Figure is available in color online only.

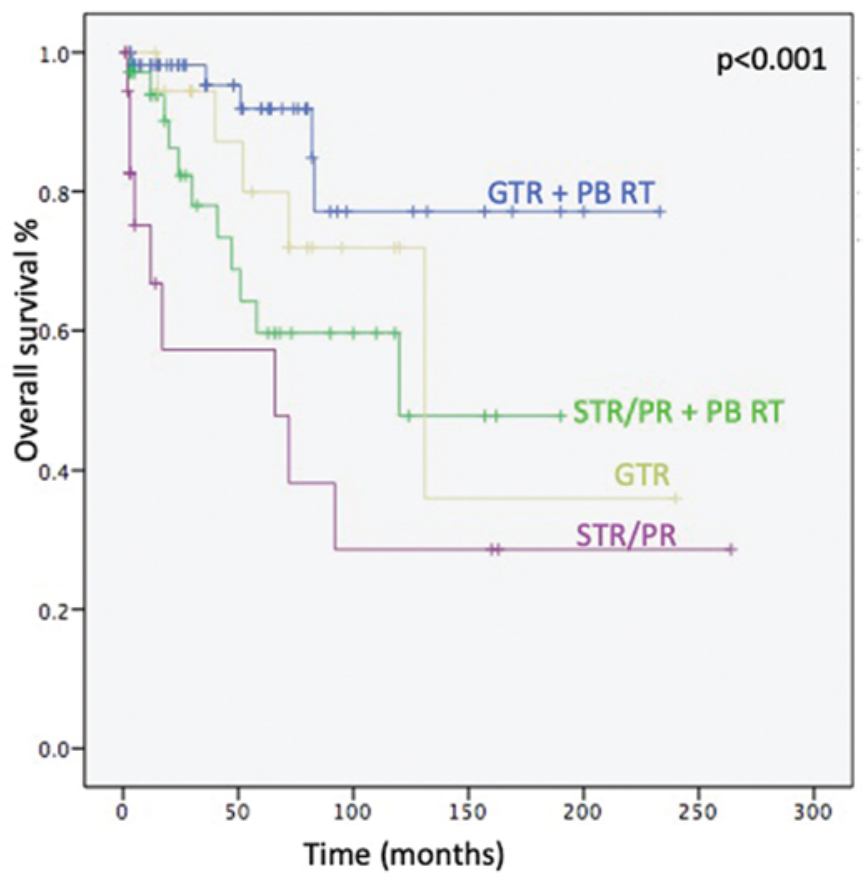


mas can be reasonably correlated with the cavernous sinus dural infiltration and invasion that was present in the majority of tumors (56 of 75 [74.7\%]).

The advantage of the EEA is not only its more direct approach to the clivus, but also the limited neurovascular morbidity associated with the procedure compared to open transcranial approaches. ${ }^{23,24,28,38}$ Moving from knowledge of the disease's aggressiveness and the need for its radical removal, we underline the problem of finding a balance between a surgical outcome that allows disease control and the need for preserving a patient's quality of life. Indeed, limiting approach morbidity is of paramount importance in clival chordoma surgery. Transcranial approaches involve a circuitous route to reach the clivus, working through spaces between cranial nerves and vascular structures, coming from a lateral to medial or superior to inferior trajectory that requires brain retraction. ${ }^{6,8,12,13}$ The likelihood of functional impairment is reduced with the transsphenoidal approach, which takes advantage of the natural nasal cavity, allowing for tumor debulking prior to reaching surrounding critical vessels and nerves and thus reducing the need for surgical maneuvers posing the risk of neurovascular damage during dissection..$^{39-42}$ Although the low numbers of postoperative complications (21 of 151) and worsening clinical and functional statuses (13 of 151 and 4 of 151 cases, respectively) in the cohort of endoscopically treated tumors do not allow for a meaningful analysis of approach-related morbidity, our results confirm the safety of the EETA for the treatment of clival chordomas. Interestingly, we observed that the number of complications related to the EETA was distributed with no significant difference among the two eras of treatment, despite the significantly increased GTR rate achieved through the EETA and the expansion of indication to middle- and lower-clival chordomas in the second period of the study. Consequently, an aggressive surgical strategy did not result in a corresponding increase in complication rates, with 10 and 11 adverse events occurring when GTR (10 of 87 [11.5\%]) and STR/PR (11 of 64 [17.2\%]) were achieved, respectively. In the present series, the most common complication associated with the EETA was CSF leakage, which is still considered the main drawback of the endoscopic technique for the removal of clival chordomas and many other skull base tumors. However, similar to previous reports, we found a low rate of CSF leakage after EETA (7 of 151 [4.6\%]), no higher than the rate after other approaches $(p=0.084)$ and with no significant differences as compared to the incidence of other complications $(\mathrm{p}=0.189)$. CSF leakage occurred in 2 upper-, 1 middle-, 2 lower-, and 2 holo-clival chordomas; as expected, all of them in clival chordomas with dural infiltration ( 2 of 7) or intradural extension (5 of 7). These results probably reflect the improvement in skull base reconstruction techniques, particularly the success of vascularized flaps..$^{24,27,35,36,40}$

Removal of clival chordomas through the EEA is probably one of the procedures that most benefits from the introduction of modern intraoperative equipment. With the assistance of neuronavigation, vascular Doppler ultrasound, and neurophysiological monitoring, it is possible to locate in advance and protect vital neurovascular structures often encroached by the tumor, which obviously decreases the complication rates. ${ }^{28,43,44}$ Our study underscores the widespread implementation of these tools in the latter era, and for the first time, we investigated their relevance to the extent of tumor removal, through both the endoscopic and other approaches. On multivariate analysis, we found the use of microvascular Doppler ultrasound to be an independent factor limiting the extent of resection. The reason for this finding is the test's reliable identification of the tumorvessel interface, which commonly precludes further safe removal of the chordomas.

Along with the evolution of the EETA, the second surgical era was marked by an increased number of GTRs. Our multivariate analysis of factors related to the extent of resection in the entire series showed that GTR is more easily achieved when an EETA is performed as compared to other approaches (87 of 151 [57.6\%] vs 6 of 31 [19.3\%]). However, we found the rate of GTR among patients operated on through the EETA (57.6\%) to be slightly inferior to rates in previous reports..$^{24,27,28}$ There are three possible reasons for this finding. First, our series encompasses an unusually high number of endoscopically treated clival chordomas with dural involvement, in terms of both infiltration (52 of 151 [34.4\%]) and intradural extension (62 of 151 [41.1\%]), which could cause an underestimation of the efficacy of the EEA..$^{11,19,25}$ Although not representing a contraindication to the endoscopic ventral route, which was used regardless of tumor extension, dural infiltration and intradural extension were limiting factors to complete tumor resection on multivariate analysis. The GTR rate was significantly higher $(\mathrm{p}<0.05)$ among endoscopically treated clival chordomas with extradural extension ( 29 of 37 [78.4\%]) than among tumors with dural infiltration (27 of 52 [51.9\%]) and intradural invasion (31 of 62 [50\%]). Indeed, in the presence of a diffusely infiltrative growth pattern, the complete removal of surrounding dura mater is not realistic; at the same time, STR represents an acceptable option when the tumor's intradural adhesion to critical neurovascular structures and the brainstem hampers the safety of an aggressive surgery. In these cases, striking the right balance between the various pros and cons of the range of approaches to the skull base, a multiple staged surgical strategy combining the endoscopic ventral route with a conventional open transcranial route may represent the best option to achieve the maximal safe resection. Another issue is the ratio of previously treated tumors in the cohort of clival chordomas removed through the EETA (52 of 151 [34.4\%]) and its impact on GTR. We found a diminished GTR rate among endoscopically treated recurrent tumors (21 of 52 [40.4\%]) compared to the rate among primary clival chordomas (66 of 99 [66.7\%]). On the basis of previous reports and our multivariate analysis, prior surgery is associated with a lower chance of radical excision, confirming the importance of striving for GTR at the primary surgery. ${ }^{7,10,14,23,27}$ One more consideration regards the multicentric design of our study, which virtually eliminates the bias of collecting and analyzing data from a single center with a high level of specialization in skull base surgery; at the same time, the recruitment of patients from different experienced centers can negatively affect the surgical outcome because of an improper learning curve in endoscopic endonasal surgery. 
Considering the entire patient cohort, on multivariate analysis, the use of EETA, GTR, and proton beam radiotherapy correlated with better survival rates. We found, similar to previous reports, histopathology to be pertinent for the prognosis of this disease: the chondroid subtype favored long-term survival compared to the dedifferentiated and classic subtypes. ${ }^{4,5}$ Proton beam therapy has been used in most of the recent series because of the unique ballistic characteristics of high-energy particles that allow dose escalation to the tumor and delivery of substantially lower doses to critical structures compared to other radiation modalities..$^{30,45}$ In this series, among 141 surgically treated clival chordomas further treated with adjuvant radiotherapy, $81.6 \%$ received proton beam radiation, with a rising trend when looking at the two treatment eras and the survival benefit compared to those with photon and Gamma Knife radiosurgery. Our Kaplan-Meier analysis of data from the whole series showed the complementary role of GTR and proton beam radiation in improving survival: patients receiving GTR had PFS and OS rates at 5 years of $65 \%$ and $70 \%$, compared to $45 \%$ and $38 \%$, respectively, when STR or PR was achieved. Patients benefit the most (90\% PFS rate and 95\% OS rate, at 5 years) when treated with a multimodal approach based on GTR and proton beam radiation.

\section{Study Limitations}

In our series, the vast majority of surgical procedures were EETAs, precluding a direct comparison with other approaches. The aim of this review was not to demonstrate the superiority of EETA, but rather to confirm its use and rapid diffusion in Italy. In point of fact, the disparity between approaches reflects the standardization of the endoscopic endonasal technique for many skull base tumors along the study period.

A second limitation is the paucity of information about radiotherapy, such as the administered dose, the exact time from the index surgery, and the patients' clinical status after it. These data could provide deeper insight into the radiation modalities, contributing to a better definition of the optimal therapeutic strategy.

Lastly, a longer follow-up is warranted for clival chordomas, which often behave as a chronic disease with a high recurrence rate over time. The follow-up in the present series is in line with the longest follow-up in the pertinent literature, with a mean of 62 months.

\section{Conclusions}

Gathering, to the best of our knowledge of the literature, the largest series of clival chordomas surgically treated through an EETA, we found an increased use of this approach among Italian neurosurgical departments together with an improvement in the extent of resection over time. The satisfactory surgical outcome achieved through the EETA in terms of GTR was marked by low surgical morbidity and the preservation of patient quality of life, and it was reinforced, in terms of PFS and OS, by the use of proton beam therapy, which was increasingly performed along the period of study. The benefit of this multimodal approach remains to be proven in a comparative prospective fashion.

\section{Acknowledgments}

This study was conducted under the auspices of SINch (Società Italiana di Neurochirurgia), Neuroendoscopy Section. For contributions to the manuscript preparation, we would like to express our appreciation to the following: Sara Bruscella, Department of Neurosurgery, "Sant'Anna e San Sebastiano" Hospital, Caserta, Italy; Alberto Campione, Division of Neurosurgery, Università degli Studi dell'Insubria, Ospedale di Circolo e Fondazione Macchi, Varese, Italy; Marco Ceraudo, Neurosurgery and Neurotraumatology, San Martino Policlinico Hospital, IRCCS for Oncology and Neurosciences, Genoa, Italy; Luci Maria Cecilia Di Mitri, Department of Pathological Anatomy, IRCCS "Casa Sollievo della Sofferenza", San Giovanni Rotondo, Italy; Giuseppe Di Perna, Department of Neuroscience "Rita Levi Montalcini", Neurosurgery Unit, University of Turin, Italy; Federico Frio, Department of Neurosurgery, IRCCS Institute of Neurological Sciences of Bologna, "Bellaria" Hospital, Bologna, Italy; Giovanbattista Lasio, Division of Neurosurgery, Humanitas Research Hospital, Rozzano, Italy; Giuseppe Oliveri, Division of Neurosurgery, AOU Senese "Le Scotte", Siena, Italy; Miran Skrap, Division of Neurosurgery, Humanitas Research Hospital, Rozzano, Italy; and Matteo Zoli, Department of Biomedical and Neuromotor Sciences, University of Bologna, IRCCS Institute of Neurological Sciences of Bologna, "Bellaria" Hospital, Bologna, Italy.

\section{References}

1. Di Maio S, Temkin N, Ramanathan D, Sekhar LN. Current comprehensive management of cranial base chordomas: 10year meta-analysis of observational studies. J Neurosurg. 2011;115(6):1094-1105.

2. George B, Bresson D, Herman P, Froelich S. Chordomas: a review. Neurosurg Clin N Am. 2015;26(3):437-452.

3. al-Mefty O, Borba LA. Skull base chordomas: a management challenge. J Neurosurg. 1997;86(2):182-189.

4. Lanzino G, Dumont AS, Lopes MB, Laws ER Jr. Skull base chordomas: overview of disease, management options, and outcome. Neurosurg Focus. 2001;10(3):E12.

5. Jones PS, Aghi MK, Muzikansky A, et al. Outcomes and patterns of care in adult skull base chordomas from the Surveillance, Epidemiology, and End Results (SEER) database. $J$ Clin Neurosci. 2014;21(9):1490-1496.

6. Tzortzidis F, Elahi F, Wright D, et al. Patient outcome at longterm follow-up after aggressive microsurgical resection of cranial base chordomas. Neurosurgery. 2006;59(2):230-237.

7. Guinto G, Guinto-Nishimura Y. Clivus chordomas: role of surgery. World Neurosurg. 2014;81(5-6):688-689.

8. Samii A, Gerganov VM, Herold C, et al. Chordomas of the skull base: surgical management and outcome. J Neurosurg. 2007;107(2):319-324.

9. Di Maio S, Rostomily R, Sekhar LN. Current surgical outcomes for cranial base chordomas: cohort study of 95 patients. Neurosurgery. 2012;70(6):1355-1360.

10. Campbell RG, Prevedello DM, Ditzel Filho L, et al. Contemporary management of clival chordomas. Curr Opin Otolaryngol Head Neck Surg. 2015;23(2):153-161.

11. Wang L, Wu Z, Tian K, et al. Clinical features and surgical outcomes of patients with skull base chordoma: a retrospective analysis of 238 patients. J Neurosurg. 2017;127(6):12571267.

12. Labidi M, Watanabe K, Bouazza S, et al. Clivus chordomas: a systematic review and meta-analysis of contemporary surgical management. J Neurosurg Sci. 2016;60(4):476-484.

13. Al-Mefty O. Chordomas: a personal perspective. In: AlMefty O, Borba L, eds. Chordomas. Thieme; 2017:179-186.

14. Stacchiotti S, Sommer J. Building a global consensus approach to chordoma: a position paper from the medical and patient community. Lancet Oncol. 2015;16(2):e71-e83.

15. Tauziéde-Espariat A, Bresson D, Polivka M, et al. Prognostic 
and therapeutic markers in chordomas: a study of $287 \mathrm{tu}-$ mors. J Neuropathol Exp Neurol. 2016;75(2):111-120.

16. Boari N, Gagliardi F, Cavalli A, et al. Skull base chordomas: clinical outcome in a consecutive series of 45 patients with long-term follow-up and evaluation of clinical and biological prognostic factors. J Neurosurg. 2016;125(2):450-460.

17. Sanusi O, Arnaout O, Rahme RJ, et al. Surgical resection and adjuvant radiation therapy in the treatment of skull base chordomas. World Neurosurg. 2018;115:e13-e21.

18. Fernandez-Miranda JC, Gardner PA, Snyderman CH, et al. Clival chordomas: a pathological, surgical, and radiotherapeutic review. Head Neck. 2014;36(6):892-906.

19. Jahangiri A, Chin AT, Wagner JR, et al. Factors predicting recurrence after resection of clival chordoma using variable surgical approaches and radiation modalities. Neurosurgery. 2015;76(2):179-185, discussion 185-186.

20. Crockard HA, Steel T, Plowman N, et al. A multidisciplinary team approach to skull base chordomas. J Neurosurg. 2001; 95(2):175-183

21. Fraser JF, Nyquist GG, Moore N, et al. Endoscopic endonasal transclival resection of chordomas: operative technique, clinical outcome, and review of the literature. J Neurosurg. 2010; 112(5):1061-1069.

22. Shimony N, Gonen L, Shofty B, et al. Surgical resection of skull-base chordomas: experience in case selection for surgical approach according to anatomical compartments and review of the literature. Acta Neurochir (Wien). 2017;159(10): $1835-1845$.

23. Sen C, Triana AI, Berglind N, et al. Clival chordomas: clinical management, results, and complications in 71 patients. $J$ Neurosurg. 2010;113(5):1059-1071.

24. Komotar RJ, Starke RM, Raper DM, et al. The endoscopeassisted ventral approach compared with open microscopeassisted surgery for clival chordomas. World Neurosurg. 2011;76(3-4):318-327, discussion 259-262.

25. Stippler M, Gardner PA, Snyderman CH, et al. Endoscopic endonasal approach for clival chordomas. Neurosurgery. 2009;64(2):268-278.

26. Rahme RJ, Arnaout OM, Sanusi OR, et al. Endoscopic approach to clival chordomas: the Northwestern experience. World Neurosurg. 2018;110:e231-e238.

27. Zoli M, Milanese L, Bonfatti R, et al. Clival chordomas: considerations after 16 years of endoscopic endonasal surgery. $J$ Neurosurg. 2018;128(2):329-338.

28. Koutourousiou M, Gardner PA, Tormenti MJ, et al. Endoscopic endonasal approach for resection of cranial base chordomas: outcomes and learning curve. Neurosurgery. 2012; 71(3):614-625.

29. Katz S, Ford AB, Moskowitz RW, et al. The index of ADL: a standardized measure of biological and psychological function. JAMA. 1963;185:914-919.

30. Amichetti M, Cianchetti M, Amelio D, et al. Proton therapy in chordoma of the base of the skull: a systematic review. Neurosurg Rev. 2009;32(4):403-416.

31. Bouche J, Guiot G, Rougerie J, Freche C. The trans-sphenoidal route in the surgical approach to chordoma of the clivus. Article in French. Ann Otolaryngol Chir Cervicofac. 1966; 83(12):817-834.

32. Hardy J. Transsphenoidal hypophysectomy. J Neurosurg. 1971;34(4):582-594.

33. Dehdashti AR, Karabatsou K, Ganna A, et al. Expanded endoscopic endonasal approach for treatment of clival chordomas: early results in 12 patients. Neurosurgery. 2008;63(2): 299-309.

34. Cappabianca P, Cavallo LM, Esposito F, et al. Extended en- doscopic endonasal approach to the midline skull base: the evolving role of transsphenoidal surgery. Adv Tech Stand Neurosurg. 2008:33:151-199.

35. Mangussi-Gomes J, Beer-Furlan A, Balsalobre L, et al. Endoscopic endonasal management of skull base chordomas: surgical technique, nuances, and pitfalls. Otolaryngol Clin North Am. 2016;49(1):167-182.

36. Chibbaro S, Cornelius JF, Froelich S, et al. Endoscopic endonasal approach in the management of skull base chordomasclinical experience on a large series, technique, outcome, and pitfalls. Neurosurg Rev. 2014;37(2):217-225.

37. Prevedello DM, Ditzel Filho LF, Solari D, et al. Expanded endonasal approaches to middle cranial fossa and posterior fossa tumors. Neurosurg Clin N Am. 2010;21(4):621-635, vi.

38. Oishi Y, Tamura R, Takahashi S, et al. A comparative study between traditional microscopic surgeries and endoscopic endonasal surgery for skull base chordomas. World Neurosurg. 2020;134:e1099-e1107.

39. Frank G, Sciarretta V, Calbucci F, et al. The endoscopic transnasal transsphenoidal approach for the treatment of cranial base chordomas and chondrosarcomas. Neurosurgery. 2006;59(1)(suppl 1):ONS50-ONS57.

40. Cappabianca P, Frank G, Pasquini E, et al. Extended endoscopic endonasal transsphenoidal approaches to the suprasellar region, planum sphenoidale and clivus. In: de Divitiis E, Cappabianca P, eds. Endoscopic Endonasal Transsphenoidal Surgery. Springer; 2003:176-187.

41. Laws ER Jr. Transsphenoidal surgery for tumors of the clivus. Otolaryngol Head Neck Surg. 1984;92(1):100-101.

42. Maira G, Pallini R, Anile C, et al. Surgical treatment of clival chordomas: the transsphenoidal approach revisited. J Neurosurg. 1996;85(5):784-792.

43. Dusick JR, Esposito F, Malkasian D, Kelly DF. Avoidance of carotid artery injuries in transsphenoidal surgery with the Doppler probe and micro-hook blades. Neurosurgery. 2007; 60(4)(suppl 2):322-329.

44. Schaberg MR, Anand VK, Schwartz TH. 10 pearls for safe endoscopic skull base surgery. Otolaryngol Clin North Am. 2010;43(4):945-954.

45. Alahmari M, Temel Y. Skull base chordoma treated with proton therapy: a systematic review. Surg Neurol Int. 2019;10: 96.

\section{Disclosures}

The authors report no conflict of interest concerning the materials or methods used in this study or the findings specified in this paper.

\section{Author Contributions}

Conception and design: Cavallo, Cappabianca. Acquisition of data: d'Avella, Mazzatenta, Catapano, Fontanella, Locatelli, Luglietto, Milani, Solari, Vindigni, Zenga, Zona. Analysis and interpretation of data: d'Avella. Drafting the article: d'Avella. Critically revising the article: Cavallo, Mazzatenta, Catapano, Fontanella, Locatelli, Luglietto, Milani, Solari, Vindigni, Zenga, Zona, Cappabianca. Approved the final version of the manuscript on behalf of all authors: d'Avella. Statistical analysis: d'Avella. Study supervision: Cavallo, Cappabianca.

\section{Correspondence}

Elena d'Avella: University of Napoli "Federico II", Naples, Italy. elenadavella@gmail.com. 